\title{
AVALIAÇÃO DO CONSUMO DE DERIVADOS DE TRIGO POR MEIO DE QUESTIONÁRIO DE FREQUÊNCIA DO CONSUMO ALIMENTAR EM MARINGÁ-PR
}

\author{
Raquel Riccomini Munhoz* \\ Magda Pires C. R. Aquino** \\ Isabelle Zanquetta Carvalho***

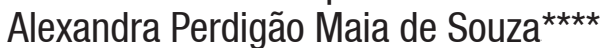

\begin{abstract}
RESUMO: 0 trigo é amplamente utilizado na alimentação humana e ocupa 0 primeiro lugar em volume de produção mundial. Esta pesquisa teve como objetivo avaliar o consumo de derivados de trigo pela população de Maringá, Paraná, e sua correlação com fatores demográficos e estado nutricional. A pesquisa foi de natureza quantitativa com coleta de dados transversal. Foram avaliados 270 indivíduos adultos com idade entre 20 a 59 anos, de ambos os sexos. Os indivíduos foram abordados nos supermercados da cidade de Maringá, Paraná. Utilizaram-se dois questionários para coleta de dados. No primeiro continham perguntas sobre 0 perfil dos indivíduos entrevistados (sexo, idade, cor, escolaridade, renda familiar, peso, estatura e Índice de Massa Corporal - IMC). No segundo continha o Questionário de Frequência Alimentar Quantitativo (QFA), utilizado para avaliar 0 consumo de derivados de trigo. As informações de peso e estatura serviram para o cálculo do IMC: peso (kg)/ estatura $(m) 2$, e então se detectou o estado nutricional segundo os pontos de corte estabelecidos pela Organização Mundial da Saúde (OMS). As associações entre o consumo de derivados de trigo e fatores demográficos e estado nutricional foram realizadas por meio de análise estatística, para a qual se utilizou o programa Epi Info versão 7.2.0.1. Os dados foram submetidos ao teste de Mann-Whitney $(p<0,05)$ para se determinar a significância dos resultados. 0s resultados demonstraram consumo maior de alimentos do tipo fast food por adultos entre $20 \mathrm{e}$ 30 anos e em quantidades expressivas em todas as faixas de IMC. Identificaram-se como derivados de trigo mais consumidos os de panificação e as massas. Não houve significância estatística ao se comparar peso com ingestão dos derivados de trigo.
\end{abstract}

PALAVRAS-CHAVE: Inquéritos nutricionais; Estado nutricional; Antropometria.

\section{THE CONSUMPTION OF WHEAT DERIVATES EVALUATED BY A QUESTIONNAIRE ON FREQUENCY OF FOOD INTAKE IN MARINGÁ}

\begin{abstract}
Wheat is not only consumed worldwide but ranks first in production. Current qualitative and transversal research assesses the consumption of wheat derivates by the population in Maringá PR Brazil, and its co-relationship with demographic factors and nutritional status. Two hundred and seventy adults, aged 20 - 59 years, males and females, were evaluated. The adults were interviewed in supermarkets in Maringá with two questionnaires for data collection. The first questionnaire dealt with the subjects' profile (gender, age, color, schooling, family wage, weight, height and Body Mass Index - BMI). The second questionnaire comprised Quantitative Food Frequency (QFF) to evaluate consumption of wheat derivates. Data on weight and height were used to calculate BMl: weight $(\mathrm{kg}) / \mathrm{height}(\mathrm{m})^{2}$, furnishing the nutrition state according to scale by WHO. Association between the intake of wheat derivates and demographic factors and nutritional state was performed by statistical analysis with Epi Info 7.2.0.1. Data underwent Mann-Whitney's test $(p<0.05)$ to determine significance of results. Results showed high consumption rate in fast food by adults aged between 20 and 30 years, and in great amounts at all BMI scales. Pastry and pasta were the most wheat derivates consumed. There was no statistical significance when weight was compared with ingestion of wheat derivates.
\end{abstract}

KEYWORDS: Nutritional survey; Nutritional status; Anthropometry.

\footnotetext{
*Acadêmico do Curso de Nutrição do Centro Universitário Cesumar - UNICESUMAR, Maringá-PR, Brasil; Bolsista PROBIC/UniCesumar.

*Acadêmico do Curso de Nutrição do Centro Universitário Cesumar - UNICESUMAR, Maringá - PR, Acadêmico colaborador no PROBIC/UniCesumar.

**** Docente orientador do Curso de Nutrição do Centro Universitário Cesumar - UNICESUMAR, Maringá-PR, Brasil.

Docente coorientador do Curso de Nutrição do Centro Universitário Cesumar - UNICESUMAR, Maringá-PR, Brasil.
} 


\section{INTRODUÇÃO}

0 trigo (Triticum aestivum L.), originário do Oriente Médio (Ásia), ocupa $20 \%$ da área mundial cultivada, e tem sido cultivado no Brasil desde 1534 pelos portugueses, sendo que o Estado do Paraná contribui com $60 \%$ da produção nacional, seguido pelos Estados do Rio Grande do Sul, Mato Grosso e São Paulo (IBGE, 2017). A produção desse grão atingiu 5,63 milhões de toneladas em 2015 no Brasil. No Paraná, estima-se que foram produzidos 3,4 milhões de toneladas. No entanto, 0 amplo consumo nacional ainda faz necessária a importação do produto (CONAB, 2017).

0 trigo é um cereal amplamente consumido e difundido na dieta humana, contribuindo com cerca de $20 \%$ do total de calorias diárias. Os derivados imediatos são a farinha branca e integral, mas há maior consumo de farinha branca, no qual seu alto conteúdo de glúten confere elasticidade às massas produzidas. Com o trigo são produzidos: pão, macarrão, bolo, biscoitos, cereais matinais, pizza, assim como triguilho para o preparo de quibe, torta de quibe, tabule e outros produtos diversos, fornecendo carboidratos complexos, bem como vitaminas do complexo B e ferro (TROMBETE et al., 2013).

A avaliação do consumo alimentar é ferramenta fundamental no processo de entendimento das associações entre alimentos e morbimortalidade, além de determinar as variáveis de influência no comportamento alimentar (MENEZES et al., 2011). Porém a mensuração do consumo de alimentos é um dos mais desafiadores aspectos da ciência e prática da nutrição e dietética, pelas limitações dos métodos para medir esta ingestão acuradamente. 0 Questionário de Frequência Alimentar (QFA) é considerado um dos principais instrumentos utilizados para coleta de dados dietéticos. 0 QFA tem como primeiro uso os estudos epidemiológicos. 0 elemento essencial do QFA é capturar a probabilidade de consumo da maioria dos alimentos, em um determinado período pregresso de tempo. Esta característica permite estudar a dieta habitual, 0 que é de fundamental importância para estimar a medida de exposição aos fatores dietéticos e investigar as possíveis associações com desfechos de interesse (FISBERG, et al., 2008).

A partir disso, o trabalho em questão buscou avaliar o consumo de derivados de trigo pela população de Maringá, Paraná (descrita segundo características demográficas) e sua correlação com fatores demográficos e estado nutricional, por meio de QFA, classificação de IMC e teste de significância por Mann-Whitney $(p<0.05)$. 0s resultados demonstraram consumo maior de alimentos do tipo fast food por adultos entre 20 e 30 anos e em quantidades expressivas em todas as faixas de IMC. Identificaramse como derivados de trigo mais consumidos os de panificação e as massas. Não houve significância estatística ao se comparar peso com ingestão dos derivados de trigo.

\section{METODOLOGIA}

A pesquisa foi de natureza quantitativa com coleta de dados transversal. Foram avaliados 270 indivíduos adultos com idade entre 20 e 59 anos, sendo 167 homens e 103 mulheres. 0s indivíduos foram abordados nos supermercados da cidade de Maringá, Paraná, no ano de 2015. 0 tamanho da amostra foi calculado conforme Barbetta (2001) utilizando a fórmula $\mathrm{n} 0=1 / \mathrm{E} 0$, em que $\mathrm{n} 0$ é a primeira aproximação para o tamanho da amostra e E0 é 0 erro amostral tolerado.

0 projeto de pesquisa foi encaminhado ao Comitê de Ética em Pesquisa (CEP) do Centro Universitário de Maringá (UniCesumar) e aprovado com Certificado de Apresentação para Apreciação Ética (CAAE), sob o parecer consubstanciado número 1.359.746.

Após aprovação, a coleta de dados foi realizada, para tanto, foram utilizados dois questionários. No primeiro continham perguntas sobre o perfil dos indivíduos entrevistados (sexo, idade, cor, escolaridade, renda familiar) e dados antropométricos de peso e estatura. No segundo continha o Questionário 
de Frequência Alimentar Quantitativo (QFA).

0 peso e estatura foram mensurados no ato da entrevista. Para a aferição do peso utilizouse uma balança digital da marca Plena ${ }^{\circledR}$, com capacidade máxima de 150 quilos. 0s participantes foram posicionados com os dois pés sobre a balança, distribuindo seu peso igualmente sobre as duas pernas, sem calçados.

Para a aferição da estatura foi utilizado um estadiômetro da marca Personal Sanny®, com escala em centímetros (cm). Os indivíduos foram avaliados sem sapatos, posicionados com os calcanhares e joelhos juntos, braços soltos e posicionados ao longo do corpo, com as palmas das mãos voltadas para as coxas, pernas retas, ombros relaxados e cabeça no plano horizontal de Frankfurt (olhando para frente, em linha reta na altura dos olhos).

As informações de peso e estatura foram utilizadas para 0 cálculo do Índice de Massa Corporal (IMC): peso(kg)/estatura $(\mathrm{m})^{2}$, e então se detectou 0 estado nutricional segundo os pontos de corte estabelecidos pela Organização Mundial da Saúde (WH0, 1997): baixo peso $\left(<18,5 \mathrm{~kg} / \mathrm{m}^{2}\right)$, peso adequado $\left(18,5-24,9 \mathrm{~kg} / \mathrm{m}^{2}\right)$, excesso de peso, $\left(\geq 25,0 \mathrm{~kg} / \mathrm{m}^{2}\right)$, pré-obesidade $\left(25,0-29,9 \mathrm{~kg} / \mathrm{m}^{2}\right)$, obesidade classe I $\left(30,0-34,9 \mathrm{~kg} / \mathrm{m}^{2}\right)$, obesidade classe II $(35,0-39,9$ $\mathrm{kg} / \mathrm{m}^{2}$ ), obesidade classe III (igual ou superior a 40,0 $\mathrm{kg} / \mathrm{m}^{2}$ ). Para fins de análise, os grupos de IMC $\geq 25 \mathrm{~kg} /$ $\mathrm{m}^{2}$ foram classificados como excesso de peso.

Foi utilizado o QFA para avaliar o consumo alimentar de trigo e de seus derivados. A quantidade dos alimentos foi avaliada considerando a porção alimentar por medida caseira para cada produto, segundo a RDC n³59 e n`360 da ANVISA (BRASIL, 2003). Calculouse a quantidade consumida de cada alimento em gramas para um período semanal a partir dos dados de tamanho da porção e frequência de consumo.

Para análise de frequência do consumo, os alimentos foram agrupados em cinco categorias: panificação (pão tipo bisnaguinha, pão francês, pão caseiro, pão de forma, torrada - tradicionais ou integrais); confeitaria (biscoitos, bolos com ou sem recheio cobertura, tortas salgadas ou doces); massas (macarrão, massas em geral, miojo); cereais (cereais matinais e barras de cereais); fast food (pastel, salgados em geral - como coxinha, esfirra, croissant - pizzas, sanduíches - hambúrguer e cachorro quente - e salgadinhos de pacote).

A média (M), primeiro quartil (Q25) e terceiro quartil (Q75) dos resultados foram determinados. As associações entre o consumo de derivados de trigo e fatores demográficos e estado nutricional foram realizadas por análise estatística, para a qual se utilizou o programa Epi Info versão 7.2.0.1. Os dados foram submetidos ao teste de Mann-Whitney $(p<0,05)$ para se determinar a significância dos resultados.

\section{RESULTADOS E DISCUSSÃO}

0 perfil sociodemográfico dos entrevistados é demonstrado na Tabela 1, sendo a maioria do gênero masculino (61,85\%). A idade média é de 35,44 $\pm 11,58$ anos, sendo $34,71 \pm 11,61$ anos para homens e 36,62 $\pm 11,49$ anos para mulheres. 0s dados diferem dos apresentados em Londrina-PR, em estudo com mesmo objetivo da presente pesquisa, onde a maior parte da amostra era do gênero feminino (SANTOS, 2009).

Quanto à escolaridade, a maioria dos entrevistados $(31,48 \%)$ apresentaram ensino superior incompleto e $24,81 \%$ apresentaram ensino médio completo. Tal perfil é similar ao demonstrado em Londrina, no qual $37 \%$ e $21,1 \%$ apresentavam ensino superior incompleto e ensino médio completo respectivamente (SANTOS, 2009). As faixas de renda mais expressivas foram de três a cinco salários mínimos (32,22\%) e cinco a dez salários mínimos (24,81\%).

Em relação ao índice de massa corporal - IMC, apenas $1,48 \%$ dos entrevistados apresentaram baixo peso, enquanto 110 indivíduos (40,74\%) apresentaram peso adequado. Dos demais participantes, verificaram -se $38,52 \%$ com pré-obesidade, $11,48 \%$ com obesidade classe I, 5,56\% com obesidade classe II e 2,22\% com obesidade classe III. Dessa forma, 0 cálculo do IMC demonstrou que $57,78 \%$ da população entrevistada apresentou excesso de peso, sendo a 
prevalência em mulheres de $69,91 \%$ e $50,3 \%$ entre os homens. A pré-obesidade $(41,75 \%)$, obesidade classe I $(21,36 \%)$ e obesidade classe II $(5,83 \%)$ foram significativamente associados com gênero feminino $(p<0,01)$. Tais resultados seguem a tendência apresentada pelo Instituto Brasileiro de Geografia e Estatística - IBGE por meio das Pesquisas de Orçamentos Familiares (POF) de 2008/2009, em que $64,9 \%$ das mulheres acima de 20 anos apresentavam excesso de peso (sendo 48,0\% com excesso de peso e $16,9 \%$ com obesidade) contra $62,5 \%$ dos homens (50,1\% com excesso de peso e 12,4\% com obesidade). Pode-se traçar um paralelo com a pesquisa realizada em Santa Catarina, na qual a prevalência de obesidade também se mostrou maior entre as mulheres (VEDANA, 2008).

Tabela 1. Perfil sociodemográfico da população entrevistada no ano de 2015 na cidade de Maringá (PR)

(continua)

\begin{tabular}{|c|c|c|c|c|c|}
\hline \multirow[t]{3}{*}{ Índices } & \multirow{2}{*}{\multicolumn{2}{|c|}{$\begin{array}{c}\text { Masculino } \\
\mathrm{N}=167\end{array}$}} & \multirow{2}{*}{\multicolumn{2}{|c|}{$\begin{array}{c}\text { Feminino } \\
\mathrm{N}=103\end{array}$}} & \multirow{3}{*}{$p^{*}$} \\
\hline & & & & & \\
\hline & FA & FR & FA & FR & \\
\hline Faixa etária/gênero & & & & & 0,14 \\
\hline 20 até 30 anos & 70 & 41,92 & 39 & 37,86 & \\
\hline 31 até 40 anos & 44 & 26,35 & 28 & 27,18 & \\
\hline 41 até 50 anos & 33 & 19,76 & 21 & 20,39 & \\
\hline 51 até 59 anos & 20 & 11,98 & 15 & 14,56 & \\
\hline Cor/gênero & & & & & 0,80 \\
\hline Branca & 118 & 70,66 & 72 & 69,90 & \\
\hline Preta & 12 & 7,19 & 6 & 5,83 & \\
\hline Amarela & 9 & 5,39 & 3 & 2,91 & \\
\hline Pardo & 25 & 14,97 & 22 & 21,36 & \\
\hline Indígena & 3 & 1,80 & - & - & \\
\hline
\end{tabular}

\begin{tabular}{lccccc}
\hline Escolaridade/gênero & & & & & 0,29 \\
\hline Fundamental incompleto & 8 & 4,79 & 4 & 3,88 & \\
Fundamental completo & 10 & 5,99 & 7 & 6,80 & \\
Ensino médio incompleto & 13 & 7,78 & 8 & 7,77 & \\
Ensino médio completo & 33 & 19,76 & 34 & 33,01 & \\
Superior incompleto & 61 & 36,53 & 24 & 23,30 & \\
Superior completo & 27 & 16,17 & 18 & 17,48 & \\
Pós-graduação & 15 & 8,98 & 8 & 7,77 &
\end{tabular}

(conclusão)

\begin{tabular}{cccc}
\hline \multirow{2}{*}{ Índices } & \multicolumn{2}{c}{ Masculino } & Feminino \\
\cline { 2 - 3 } & \multicolumn{2}{c}{$\mathrm{N}=167$} & $\mathrm{~N}=103$ \\
\hline & FA & FR & FA $\quad$ FR \\
\hline
\end{tabular}

\begin{tabular}{|c|c|c|c|c|c|}
\hline Renda familiar/gênero & & & & & 0,37 \\
\hline Até 1 salário mínimo & 1 & 0,60 & 3 & 2,91 & \\
\hline $\begin{array}{l}\text { Mais de } 1 \text { a } 2 \text { salários } \\
\text { mínimos }\end{array}$ & 20 & 11,98 & 6 & 5,83 & \\
\hline $\begin{array}{l}\text { Mais de } 2 \text { a } 3 \text { salários } \\
\text { mínimos }\end{array}$ & 37 & 22,16 & 20 & 19,42 & \\
\hline $\begin{array}{l}\text { Mais de } 3 \text { a } 5 \text { salários } \\
\text { mínimos }\end{array}$ & 51 & 30,54 & 36 & 34,95 & \\
\hline $\begin{array}{l}\text { Mais de } 5 \text { a } 10 \text { salários } \\
\text { mínimos }\end{array}$ & 41 & 24,55 & 26 & 25,24 & \\
\hline $\begin{array}{l}\text { Mais de } 10 \text { a } 15 \text { salários } \\
\text { mínimos }\end{array}$ & 8 & 4,79 & 4 & 3,88 & \\
\hline $\begin{array}{l}\text { Mais de } 15 \text { a } 20 \text { salários } \\
\text { mínimos }\end{array}$ & 5 & 2,99 & 4 & 3,88 & \\
\hline $\begin{array}{l}\text { Mais de } 20 \text { a } 30 \text { salários } \\
\text { mínimos }\end{array}$ & 3 & 1,80 & 4 & 3,88 & \\
\hline $\begin{array}{l}\text { Mais de } 30 \text { salários } \\
\text { mínimos }\end{array}$ & 1 & 0,60 & - & - & \\
\hline IMC/gênero & & & & & $<0,01$ \\
\hline Baixo peso & 4 & 2,40 & - & - & \\
\hline Peso adequado & 79 & 47,31 & 31 & 30,10 & \\
\hline Pré-obesidade & 61 & 36,53 & 43 & 41,75 & \\
\hline Obesidade classe I & 9 & 5,39 & 22 & 21,36 & \\
\hline Obesidade classe II & 9 & 5,39 & 6 & 5,83 & \\
\hline Obesidade classe III & 5 & 2,99 & 1 & 0,97 & \\
\hline
\end{tabular}

$\mathrm{N}=$ número; $F A=$ frequência absoluta; $F R=$ frequência relativa; IMC= índice de massa corporal; ‘Teste qui-quadrado.

Ao analisar-se a média de consumo por faixa etária, verificou-se que 0 grupo de 20 a 30 anos apresentou consumo mais elevado de massas, cereais e fast food, em contraste com as demais faixas etárias $(p<0,05)$, (Tabela 2). É importante destacar a alta ingestão de alimentos do tipo fast-food, uma vez que a dieta brasileira demonstra um acréscimo na introdução de alimentos processados de alta densidade calórica, padrão de consumo compatível com os altos níveis de indivíduos com excesso de peso e desordens metabólicas no país (SOUZA, 2013). Tal consumo é expressivo em todas as faixas 
etárias, apesar de se verificar decréscimo na média conforme aumento da idade. A ingestão de produtos de confeitaria demonstrou-se praticamente equivalente nos grupos de indivíduos de 20 até 30 anos e de 31 até 40 anos e também tende a diminuir nas faixas etárias mais elevadas. Além disso, verificou-se que produtos de panificação são consumidos por todos os grupos etários em quantidades médias semelhantes.

A Tabela 3 relaciona 0 consumo de derivados de trigo com a renda familiar dos entrevistados. É possível

Tabela 2. Média de consumo semanal por faixa etária

\begin{tabular}{|c|c|c|c|c|c|c|}
\hline Faixa Etária & N & $\begin{array}{l}\text { Panificação } \\
{ }^{*} p=0,8096\end{array}$ & $\begin{array}{l}\text { Confeitaria } \\
p=0,0093\end{array}$ & $\begin{array}{l}\text { Massas } \\
p=0,0255\end{array}$ & $\begin{array}{l}\text { Cereais } \\
p=0,0799\end{array}$ & $\begin{array}{l}\text { Fast Food } \\
p=0,0002\end{array}$ \\
\hline 20 até 30 & 109 & $\begin{array}{c}397,2^{\mathrm{a}} \\
\left(204^{\mathrm{b}}-700^{\mathrm{c}}\right)\end{array}$ & $\begin{array}{c}146 \\
(60,67-284,67)\end{array}$ & $\begin{array}{c}285 \\
(200-459,5)\end{array}$ & $\begin{array}{c}17,5 \\
(0-145)\end{array}$ & $\begin{array}{c}255,5 \\
(113,1667-476)\end{array}$ \\
\hline 31 até 40 & 72 & $\begin{array}{c}367,6 \\
(237-700)\end{array}$ & $\begin{array}{c}149 \\
(60-327,5)\end{array}$ & $\begin{array}{c}200 \\
(193,3333-400)\end{array}$ & $\begin{array}{c}0 \\
(0-89,5)\end{array}$ & $\begin{array}{c}240 \\
(125,5-482,5)\end{array}$ \\
\hline 41 até 50 & 54 & $\begin{array}{c}387,6667 \\
(209,27-700)\end{array}$ & $\begin{array}{c}77,75 \\
(28-165,73)\end{array}$ & $\begin{array}{c}200 \\
(140-400)\end{array}$ & $\begin{array}{c}0 \\
(0-85)\end{array}$ & $\begin{array}{c}118 \\
(56-339,5)\end{array}$ \\
\hline 51 até 60 & 35 & $\begin{array}{c}400 \\
(350-712,6)\end{array}$ & $\begin{array}{c}45,7333 \\
(23,3333-214,6667)\end{array}$ & $\begin{array}{c}200 \\
(19,8333-285)\end{array}$ & $\begin{array}{c}0 \\
(0-33,8333)\end{array}$ & $\begin{array}{c}125 \\
(14-195)\end{array}$ \\
\hline
\end{tabular}

Os valores são apresentados como mediana ${ }^{\mathrm{a}}\left(1^{0}\right.$ quartil ${ }^{\mathrm{b}}$ e $3^{0}$ quartilc). ${ }^{*}$ Teste de Mann-Whitney

perceber que a ingestão de produtos de panificação é mais expressiva no grupo de rendimento familiar de até um salário mínimo, apesar de não haver significância na análise estatística. Os resultados demonstram semelhança na média de consumo entre indivíduos com renda de um a dois e de dois a três salários mínimos, bem como entre indivíduos com renda de três a cinco e de cinco a dez salários mínimos, para todos os grupos de alimento. Além disso, verificou-se que a média semanal de ingestão de massas é análoga em todas as faixas de rendimento, exceto na de dez a 15 salários mínimos, na qual se mostrou maior, e na de 20 a 30 salários mínimos, na qual foi menor. Em Londrina, identificou-se 0 macarrão como produto mais consumido semanalmente pelos entrevistados (SANTOS et al., 2009). A presente pesquisa demonstrou que as massas são produtos frequentes na mesa dos habitantes de Maringá, entretanto os produtos de panificação são os mais consumidos. Em Brasília, identificou-se um elevado consumo semanal de pães e massas por $89,6 \%$ e $79 \%$ dos entrevistados, respectivamente (MARINHO; HAMANN; LIMA, 2007).

Algumas pesquisas realizadas no Brasil e em outros países revelam uma relação inversamente proporcional entre 0 consumo de alimentos ricos em carboidratos e a renda dos indivíduos. Aponta-se que a participação de grupos de alimentos proteicos tende a aumentar uniformemente com 0 nível de rendimento familiar (LEVY et al., 2012). Contudo, os dados obtidos em Maringá não demonstraram diferença estatisticamente relevante entre os grupos de rendimento avaliados, exceto no tocante ao consumo de cereais que apresentou maior consumo no grupo de rendimento familiar de até um salário mínimo, $(p=0,0326)$.

Quanto ao IMC, as análises não demonstraram significância estatística que relacione 0 peso com 0 consumo de derivados de trigo (Tabela 4). Todavia, 
Tabela 3. Média de consumo semanal por renda familiar

\begin{tabular}{|c|c|c|c|c|c|c|}
\hline Renda Familiar & $\mathbf{N}$ & $\begin{array}{l}\text { Panificação } \\
{ }^{*} p=0,5388\end{array}$ & $\begin{array}{l}\text { Confeitaria } \\
\qquad p=0,0571\end{array}$ & $\begin{array}{l}\text { Massas } \\
p=0,6169\end{array}$ & $\begin{array}{l}\text { Cereais } \\
p=0,0326\end{array}$ & $\begin{array}{l}\text { Fast Food } \\
p=\mathbf{0 , 2 0 3 2}\end{array}$ \\
\hline $\begin{array}{l}\text { Até } 1 \text { salário } \\
\text { mínimo }\end{array}$ & 4,00 & $\begin{array}{c}1078,00^{\mathrm{a}} \\
\left(375^{\mathrm{a}}-1470^{\mathrm{b}}\right)\end{array}$ & $\begin{array}{c}255,4167 \\
(152,916-279)\end{array}$ & $\begin{array}{c}246,67 \\
(46,67-627,5)\end{array}$ & $\begin{array}{c}140,00 \\
(55,42-332,5)\end{array}$ & $\begin{array}{c}605,25 \\
(140,75-961,5)\end{array}$ \\
\hline $\begin{array}{l}\text { Mais de } 1 \text { a } 2 \\
\text { salário mínimo }\end{array}$ & 26,00 & $\begin{array}{c}418,00 \\
(350-700)\end{array}$ & $(41,3333-294)$ & $\begin{array}{c}200,00 \\
(186,67- \\
439,67)\end{array}$ & $(0-16,33)$ & $\begin{array}{c}134,17 \\
(86,33-374)\end{array}$ \\
\hline $\begin{array}{l}\text { Mais de } 2 \text { a } 3 \\
\text { salário mínimo }\end{array}$ & 57,00 & $\begin{array}{c}412,00 \\
(250-700)\end{array}$ & $\begin{array}{c}116,33 \\
(45,5-260,166)\end{array}$ & $\begin{array}{c}200,00 \\
(85-400)\end{array}$ & $\begin{array}{c}0,00 \\
(0-35)\end{array}$ & $\begin{array}{c}156,33 \\
(58,33-292,67)\end{array}$ \\
\hline $\begin{array}{l}\text { Mais de } 3 \text { a } 5 \\
\text { salário mínimo }\end{array}$ & 87,00 & $\begin{array}{c}420,00 \\
(200-756)\end{array}$ & $\begin{array}{c}106,00 \\
(25-265)\end{array}$ & $\begin{array}{c}200,00 \\
(200-400)\end{array}$ & $\begin{array}{c}0,00 \\
(0-105)\end{array}$ & $\begin{array}{c}240,00 \\
(86,33-476)\end{array}$ \\
\hline $\begin{array}{l}\text { Mais de } 5 \text { a } 10 \\
\text { salário mínimo }\end{array}$ & 67,00 & $\begin{array}{c}350,00 \\
(191,33-700)\end{array}$ & $\begin{array}{c}91,00 \\
(52,33-194,6)\end{array}$ & $\begin{array}{c}200,00 \\
(186,67- \\
439,67)\end{array}$ & $\begin{array}{c}0,00 \\
(0-84)\end{array}$ & $\begin{array}{c}232,00 \\
(70-371)\end{array}$ \\
\hline $\begin{array}{l}\text { Mais de } 10 \text { a } 15 \\
\text { salário mínimo }\end{array}$ & 12,00 & $\begin{array}{c}293,00 \\
(149-491,3)\end{array}$ & $\begin{array}{c}104,00 \\
(18,67-192,57)\end{array}$ & $\begin{array}{c}342,50 \\
(170-400)\end{array}$ & $\begin{array}{c}120,00 \\
(0-542,5)\end{array}$ & $\begin{array}{c}269,58 \\
(0-490)\end{array}$ \\
\hline $\begin{array}{l}\text { Mais de } 15 \text { a } 20 \\
\text { salário mínimo }\end{array}$ & 9,00 & $\begin{array}{c}350,00 \\
(350-450)\end{array}$ & $\begin{array}{c}380,00 \\
(185,67-420)\end{array}$ & $\begin{array}{c}200,00 \\
(200-285)\end{array}$ & $\begin{array}{l}25,00 \\
(0-95)\end{array}$ & $\begin{array}{c}365,00 \\
(199,5-480)\end{array}$ \\
\hline $\begin{array}{l}\text { Mais de } 20 \text { a } 30 \\
\text { salário mínimo }\end{array}$ & 7,00 & $\begin{array}{c}349,20 \\
(150-777,4667)\end{array}$ & $\begin{array}{c}320,00 \\
(16,33-369,5)\end{array}$ & $\begin{array}{c}140,00 \\
(0-400)\end{array}$ & $\begin{array}{c}5,83 \\
(0-270)\end{array}$ & $\begin{array}{c}168,00 \\
(116-772)\end{array}$ \\
\hline $\begin{array}{c}\text { Mais de } 30 \\
\text { salário mínimo }\end{array}$ & 1,00 & $(473,6-473,6)$ & $(548,5-548,5)$ & $\begin{array}{c}(285,83- \\
285,83)\end{array}$ & $(124,5-124,5)$ & $(815-815)$ \\
\hline
\end{tabular}

Os valores são apresentados como mediana a ${ }^{\left({ }^{\circ}\right.}$ quartil ${ }^{\mathrm{b}}$ e $3^{0}$ quartilc $)$. ${ }^{*}$ Teste de Mann-Whitney

é importante destacar que a ingestão de cereais só foi relevante em indivíduos que apresentaram peso adequado. Alimentos pertencentes ao grupo, como barras de cereais, são associados à alimentação saudável (FREITAS; MORETTI, 2006).

Diferentemente do esperado, observou-se ingestão de derivados de trigo menor ou equivalente entre indivíduos que apresentaram excesso de peso em comparação com os demais grupos. Sabe-se que o QFA é um instrumento de boa validade e reprodutibilidade, entretanto não está isento de erros. A coleta de dados realizada por entrevistadores capacitados e treinados minimizou vícios de classificação, contudo é preciso considerar limitações do método, uma vez que depende da memória e da capacidade do entrevistado de relatar os alimentos e porções consumidos (SELEM et al., 2014). Além disso, sabe-se que a obesidade é uma 
doença de etiologia complexa e de caráter multifatorial. 0 aumento de fornecimento de energia pela dieta $\mathrm{e}$ a redução da atividade física favorecem 0 excesso de peso ao gerar um quadro de balanço energético positivo, todavia é necessário levar em conta os fatores genéticos, metabólicos, socioculturais e psíquicos, que envolvem a obesidade e as comorbidades associadas (WANDERLEY; FERREIRA, 2010).

É importante salientar que as estratégicas de prevenção a doenças crônicas não transmissíveis no Brasil incluem a alimentação saudável para todos os grupos etários como fundamental, independentemente do índice de massa corporal apresentado pelo indivíduo (MALTA; MORAIS NETO; SILVA JUNIOR, 2011). 0 Dietary Guidelines for Americans, publicado em 2015, considera 0 consumo de limitado de alimentos ricos em carboidratos simples, gorduras saturadas e sódio um importante passo para a construção de uma alimentação saudável. Dessa forma, alimentos do tipo fast-food deveriam ter consumo reduzido em todos os grupos estudados.

Tabela 4. Média de consumo semanal por IMC

\begin{tabular}{ccccccc}
\hline IMC & $\mathbf{N}$ & $\begin{array}{c}\text { Panificação } \\
{ }^{*} \boldsymbol{p}=\mathbf{0 , 5 9 6 5}\end{array}$ & $\begin{array}{c}\text { Confeitaria } \\
\boldsymbol{p}=\mathbf{0 , 9 2 8 2}\end{array}$ & $\begin{array}{c}\text { Massas } \\
\boldsymbol{p}=\mathbf{0 , 5 1 3 4}\end{array}$ & $\begin{array}{c}\text { Cereais } \\
\boldsymbol{p}=\mathbf{0 , 0 6 7 9}\end{array}$ & $\begin{array}{c}\text { Fast Food } \\
\boldsymbol{p}=\mathbf{0 , 2 9 7 6}\end{array}$ \\
\hline \multirow{2}{*}{ Baixo peso } & 4 & $379,00^{\mathrm{a}}$ & 127,87 & 300,00 & 0,00 & 268,50 \\
& & $\left(200^{\mathrm{b}}-729^{\mathrm{c}}\right)$ & $(46-204,1)$ & $(200-500)$ & $(0-25)$ & $(137,08-341)$ \\
Peso adequado & \multirow{2}{*}{110} & 397,33 & 104,57 & 200,00 & 12,83 & 240,00 \\
& & $(261,67-700)$ & $(42-277,9)$ & $(186,67-400)$ & $(0-124,5)$ & $(113,17-480)$ \\
Excesso de peso & \multirow{2}{*}{156} & 374,13 & 120,50 & 200,00 & 0,00 & 184,67 \\
& & $(200-700)$ & $(38,5-254,22)$ & $(139,42-400)$ & $(0-84)$ & $(70-379,92)$
\end{tabular}

Os valores são apresentados como mediana a $\left(1^{\circ}\right.$ quartil ${ }^{\mathrm{b}}$ e $3^{\circ}$ quartilc $)$. ${ }^{*}$ Teste de Mann-Whitney

\section{CONSIDERAÇÕES FINAIS}

Pode-se concluir ao fim da pesquisa que 0 grupo estudado apresentou alta prevalência de excesso de peso, principalmente entre as mulheres. Verificouse um alto consumo de produtos de panificação e de massas em comparação com todas as faixas etárias, rendimentos familiares e índices de massa corporal. As análises não demonstraram significância estatística que associe 0 consumo de derivados de trigo com 0 peso. Entretanto, nota-se um consumo elevado de fast food em todos os grupos de IMC. Sabe-se que a alimentação saudável é importante fator de prevenção de obesidade e suas comorbidades. Portanto, alimentos do tipo fast food deveriam ter 0 consumo reduzido no grupo estudado.

\section{REFERÊNCIAS}

BARBETTA, P. B. Estatística aplicada às ciências sociais. 4. ed. Florianópolis: Ed. da UFSC, 2001.

BRASIL. Resolução RDC n.359, de 23 de dezembro de 2003. A Diretoria Colegiada da ANVISA/MS aprova 0 regulamento técnico de porções de alimentos embalados para fins de rotulagem nutricional. Diário Oficial da União. v.251, p.28, Seção 1, 2003.

BRASIL. Resolução RDC n.360, de 23 de dezembro de 2003. A Diretoria Colegiada da ANVISA/MS aprova o regulamento técnico sobre rotulagem nutricional de alimentos embalados. Diário Oficial da União, v.251, p.33, Seção 1, 2003. 
CONAB. Companhia Nacional de Abastecimento. Acompanhamento da safra brasileira. 2009. Disponível em: <http://www.conab.gov.br/0lalaCMS/uploads/ arquivos/15_12_11_11_02_58_boletim_graos_ dezembro_2015.pdf>. Acessado: 03 jan. 2017.

FISBERG, R. M. et al. Questionário de frequência alimentar para adultos com base em estudo populacional. Rev Saúde Pública, v. 42, n. 3, p. 550554, 2008.

FREITAS, D.G.C.; MORETTI, R.H. Caracterização e avaliação sensorial de barra de cereais. Revista Ciência e Tecnologia de Alimentos, v. 26, n. 2, p.318-324, 2006.

IBGE. Instituto Brasileiro de Geografia e Estatística. Pesquisa de orçamentos familiares 2008-2009. Aquisição alimentar domiciliar per capita brasil e grandes regiões. 2010. Disponível em: <http:// www.ibge.gov.br/home/estatistica/populacao/ condicaodevida/pof/2008_2009_aquisicao/default. htm >. Acesso: 03 jan. 2017.

LEVY, R.B.; CLARO, R.M; MONDINI, L.; SICHIERI, R.; MONTEIRO, C.A. Regional and socioeconomic distribution of household food availability in Brazil, in 2008-2009. Rev Saude Publica, v. 46, p.6-15, 2012.

MALTA, D.C.; MORAIS NETO, O.L.; SILVA JUNIOR, J.B. Apresentação do plano de ações estratégicas para 0 enfrentamento das doenças crônicas não transmissíveis no Brasil, 2011 a 2022. Epidemiologia e Serviços de Saúde. v.20, n.4, p.425-438, 2011.

MARINHO, M.C.S.; HAMANN, E.M.; LIMA, A.C.C.F. Práticas e mudanças no comportamento alimentar na população de Brasília, Distrito Federal, Brasil. Rev. Bras. Saúde Matern. Infant., Recife, v. 7, n. 3, p. 251261, 2007.

MENEZES, M.C.; HORTA, P.M.; SANTOS, L.C.; LOPES. A.C.S. Avaliação do consumo alimentar e de nutrientes no contexto da atenção primária à saúde. Ceres. v.6, n.3, p.175-190, 2011.
SANTOS, J.S.; OLIVEIRA, T.M.; PIZA FILHO, J.T.; ONO, E.Y.S.; HIROOKA, E.Y.Avaliação da ingestão de derivados de trigo em Londrina, PR, por meio de questionário de frequência de consumo de alimentos. Nutrire: Revista da Sociedade Brasileira de Alimentação e Nutrição, v.34, n.3, p.153-164, 2009.

SELEM, S.S.; CARVALHO, A.M.; VERLY-JUNIOR, E.; CARLOS, J.V.; TEIXEIRA, J.A.; MARCHIONI, D.M. Validity and reproducibility of a food frequency questionnaire for adults of Sao Paulo, Brazil. Revista brasileira de epidemiologia, v.17, n.4, p.852-859, 2014.

SOUZA, A.D.E.M.; PEREIRA, R.A.; YOKO0, E.M.; LEVY, R.B.; SICHIERI, R. Most consumed foods in Brazil: National Dietary Survey 2008-2009. Rev Saude Publica, v.47, suppl. 1, p.190s-199s, 2013.

TROMBETE, F. Aflatoxinas y tricotecenos en trigo y derivados: incidencia de la contaminación y métodos de determinación. Revista chilena de nutrición, v. 40, n. 2, p. 181-188, 2013.

U.S. DEPARTMENT OF HEALTH AND HUMAN SERVICES AND U.S. DEPARTMENT OF AGRICULTURE. 20152020. Dietary Guidelines for Americans. 8th ed. December 2015. Disponível em: <http://health.gov/ dietaryguidelines/2015/guidelines/>. Acesso: $20 \mathrm{fev}$. 2017.

VEDANA, E.H.B.; PERES, M.A.; NEVES, J.; ROCHA, G.C.; LONGO, G.Z. Prevalência de obesidade e fatores potencialmente causais em adultos em região Sul do Brasil. Arq Bras Endocrinol Metab., v. 52, p. 11561162, 2008

WANDERLEY, E.N.; FERREIRA, V.A. Obesidade: uma perspectiva plural. Ciênc Saúde Col., v.15, n.1, p.18594, 2010.

WORLD HEALTH ORGANIZATION. Obesity: preventing and managing the global epidemic. Geneva: World Health Organization; 1997.

Recebido em: 23 de fevereiro de 2017 Aceito em: 03 de maio de 2017 